\title{
PERFIL SOCIODEMOGRÁFICO DE IDOSOS VÍTIMAS DE SUICÍDIO EM UM ESTADO DO NORDESTE DO BRASIL
}

\author{
SOCIODEMOGRAPHIC PROFILE OF ELDERLY SUICIDE \\ VICTIMS IN A NORTHEASTERN STATE OF BRAZIL
}

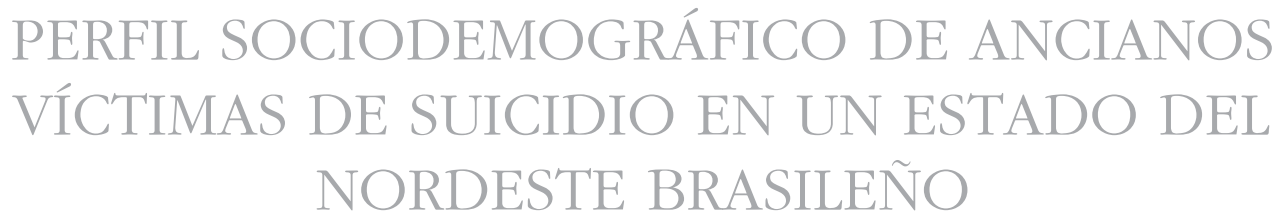

Adriana Vasconcelos Gomes ${ }^{1}$ Prissilla Kalyne Bezerra Cardoso ${ }^{2}$ Francisca Cecília Viana Rocha ${ }^{3}$ Cláudia Maria Sousa de Carvalho ${ }^{4}$ Magda Coeli Vitorino Sales ${ }^{5}$

Como citar este artigo: Gomes AV, Cardoso PKB, Rocha FCV, Carvalho CMS, Sales MCV. Perfil sociodemográfico de idosos vítimas de suicídio em um estado do Nordeste do Brasil. Rev baiana enferm. 2018;32:e26078.

Objetivo: caracterizar o perfil do idoso vítima de suicídio e identificar os meios utilizados para cometer o suicídio. Método: estudo epidemiológico, descritivo, realizado em um Instituto de Medicina Legal de referência. A amostra foi constituída de 61 declarações de óbito por suicídio ocorridos no período entre 2007 e 2014. Para a coleta, utilizou-se o questionário. Os dados foram tratados por meio de análise estatística descritiva. Resultados: o perfil do idoso que cometeu suicídio foi o de homens, com baixa escolaridade, aposentados, casados e residentes na capital do estado. Domicílio e enforcamento foram local e meio frequentes. Conclusão: sexo, escolaridade, estado civil, situação laboral e área de moradia foram as principais variáveis que caracterizaram o perfil dos idosos que cometeram suicídio. O meio físico (enforcamento) foi a principal estratégia utilizada, e o espaço privado (o domicílio) foi o local de escolha para cometer o ato.

Descritores: Mortalidade. Idoso. Suicídio.

Objective: characterize the profile of elderly suicide victims and identify the method used for committing suicide. Method: descriptive epistemological study conducted at an Institute of Legal Medicine of reference. The sample was comprised of 61 death certificates for suicides that occurred between 2007 and 2014. A questionnaire was used for the data collection. The data was treated through a descriptive statistical analysis. Results: the profile of elderly people who committed suicide had the following characteristics: men, low level of education, retired, married and state capital residents. The most frequent location and method was the home, by hanging. Conclusion: sex, level of education, marital status, employment status and location of residence were the main variables that characterized the profile of the elderly people who had committed suicide. A physical method (hanging) was the main strategy used and a private location (the home) was the place of choice for committing the act.

Keywords: Mortality. Aged. Suicide.

\footnotetext{
Enfermeira. Especialista em Saúde da Família. Teresina, Piauí, Brasil. adriannavgomes@gmail.com

Enfermeira. Especialista em Terapia Intensiva. Teresina, Piaú, Brasil.

Enfermeira. Mestre em Enfermagem. Docente do Centro Universitário Uninovafapi. Teresina, Piauí, Brasil.

Enfermeira. Mestre em Políticas Públicas. Docente do Centro Universitário Uninovafapi. Teresina, Piauí, Brasil.

Enfermeira. Mestre em Saúde da Família. Docente do Centro Universitário Uninovafapi. Teresina, Piaú́, Brasil.
} 
Objetivo: caracterizar el perfil del anciano víctima de suicidio e identificar los medios utilizados para cometer el acto. Método: estudio epidemiológico, descriptivo, en Instituto de Medicina Legal de referencia. Muestra constituida de 61 declaraciones de muerte por suicidio ocurridas entre 2007 y 2014. Para recolección, se utilizó el cuestionario. Datos tratados por medio de análisis estadístico descriptivo. Resultados: el perfil del anciano que cometió suicidio fue de hombres, con baja escolaridad, jubilados, casados y residentes en la capital del Estado. Domicilio y aborcadura fueron locales y medio frecuentes. Conclusión: sexo, escolaridad, estado civil, situación laboral y área de vivienda fueron las principales variables que caracterizaron el perfil de ancianos que cometieron suicidio. El medio físico (ahorcamiento) fue la principal estrategia utilizada, y el espacio privado (domicilio) fue el lugar de elección para cometer el acto.

Descriptores: Mortalidad. Anciano. Suicidio.

\section{Introdução}

Projeções populacionais da Organização das Nações Unidas (ONU) indicam que, até o ano de 2050, o número mundial de pessoas idosas poderá atingir cerca de 2,1 bilhões. No Brasil, a porcentagem de pessoas idosas pode aumentar de 12\% em 2015 para 29\% em 2050. Esse processo de transição sociodemográfica requer políticas públicas para enfrentá-la ${ }^{(1)}$.

O envelhecimento compreende uma fase do desenvolvimento humano que abrange fenômenos biológicos e psicossociais da vida do idoso. Limitações físicas, enfermidades, bem como a autopercepção da saúde e a perda de autonomia podem gerar sentimentos de frustação, inutilidade e perda da dignidade, culminando em sofrimento psíquico que compromete a qualidade de vida. Desta forma, as mudanças decorrentes do processo de envelhecimento podem ser fatores de risco de comportamento suicida ${ }^{(2)}$.

O suicídio é um emergente problema de saúde pública, pois mais de 800 mil pessoas antecipam a própria morte em todo o mundo ${ }^{(3)}$. O Brasil encontra-se entre os dez países com maiores registros de suicídios; na população idosa, as taxas variam entre 6,8/100.000 hab. em 2000 e 8,0/100.000 hab. em $2007^{(4)}$. Na última década, o Nordeste apresentou crescimento percentual de $72,4 \%$ na taxa de suicídio, o maior em relação às demais regiões brasileiras ${ }^{(5)}$.

O Ministério da Saúde, pela Portaria n. 1.876, de 14 de agosto de 2006, ressalta a importância dos cuidados integrais, por meio da promoção, prevenção, tratamento e recuperação da saúde, bem como a identificação de determinantes e condicionantes do suicídio e/ou tentativas, além da capacitação dos profissionais da atenção primária para o adequado conhecimento e manejo dos fatores envolvidos nos processos de suicídio $^{(6)}$.

Nesse contexto, o evidente crescimento populacional de idosos torna essencial reconhecer e preparar-se para as demandas pessoais e coletivas, relacionadas aos aspectos biológicos e socioambientais que advêm com a idade. Por isso, é relevante para os gestores e profissionais de saúde aprofundar e reconhecer aspectos envolvidos com o suicídio nesse grupo, a fim de considerá-los na definição de políticas públicas e na prestação de cuidados à saúde mais resolutivos e que tenham o caráter preventivo, com valorização do idoso, na perspectiva da integralidade da atenção, para assegurar a qualidade de vida desse grupo populacional.

Neste estudo, objetiva-se caracterizar o perfil do idoso vítima de suicídio e identificar os meios utilizados para cometer o suicídio.

\section{Método}

Trata-se de um estudo epidemiológico, retrospectivo, de caráter exploratório-descritivo e abordagem quantitativa, realizado no Instituto de Medicina Legal (IML) situado no município de Teresina, referência para o estado do Piauí, Brasil. Ressalta-se que, nos casos de óbito que não ocorrem no contexto hospitalar, as Declarações de Óbito (DO) são emitidas pelo IML justificando a escolha do cenário. 
A amostra foi obtida por meio do acesso às Declarações de Óbito de pessoas idosas com o registro da causa da morte como suicídio. Foram considerados como suicídios os óbitos por lesões autoprovocadas voluntariamente (X60-X84, de acordo com o CID-10). O instrumento utilizado para realizar a coleta de dados foi um questionário construído pelas pesquisadoras com base nas informações contidas na DO, instrumento de registro dos óbitos no território brasileiro, conforme a Lei n. 6.015/1973, que dispõe sobre os registros públicos ${ }^{(7)}$.

O questionário foi organizado por numeração ordinal e dispôs de 10 itens divididos em dois blocos: o primeiro, com as variáveis faixa etária, sexo, raça/cor, estado civil, escolaridade, ocupação e município de residência; o segundo bloco considerou as variáveis município de ocorrência, local de ocorrência do óbito e meio utilizado para efetivação do suicídio.

O levantamento de dados foi realizado pelas pesquisadoras durante os meses de julho e agosto de 2015 em sala reservada da instituição coparticipante, a fim de manter o sigilo das informações. Atendendo aos critérios pré-estabelecidos, foram incluídas no estudo, as declarações de óbito de pessoas de ambos os sexos com idade igual ou superior a 60 anos que tivessem cometido suicídio entre os anos de 2007 e 2014. Foram excluídas as declarações de óbito com dados incompletos ou ilegíveis.

Foram incluídas 61 declarações de óbito por suicídio de idosos no estado do Piauí. Os dados foram organizados em planilha do Excel for Windows com dupla digitação. Posteriormente foram processados no software Statistical Package for Social Science (SPSS versão 20.0). A análise deu-se por emprego de estatística descritiva, organizando-se os dados numéricos em tabelas de distribuição de frequência absoluta e percentuais.

Ressalta-se que, obedecendo às determinações da Resolução n. 466, de dezembro de 2012, do Conselho Nacional de Saúde ${ }^{(8)}$, o estudo somente foi iniciado após a autorização da instituição coparticipante e aprovação do projeto por um Comitê de Ética em Pesquisa ( $\mathrm{Pa}-$ recer n. 1084760, em 28 de maio de 2015, CAAE: 45191615.6.0000.5210).

\section{Resultados}

No período de 2007 a 2014, ocorreram 79 óbitos por suicídio na faixa etária de 60 anos ou mais no estado do Piauí. Desse total foram eliminados 18 casos, em virtude de incompletude, de forma simultânea, das seguintes informações: raça/cor; estado civil e escolaridade.

Assim, as 61 DOs que compuseram a amostra revelaram que os casos de suicídio de idosos no estado, ocorridos no período estudado, apresentaram incidência crescente a partir do ano de 2010. Percebeu-se uma rápida e contínua ascensão de mortes autoprovocadas, atingindo o ápice de ocorrências no ano de 2013, com 28\% dos casos. Em relação aos anos anteriores, o ano de 2014 apresentou a menor taxa dos casos notificados, 10\% do número total de casos (Gráfico 1).

Gráfico 1 - Incidência de suicídio de idosos. Piauí, Brasil - 2007-2014 (N=61)

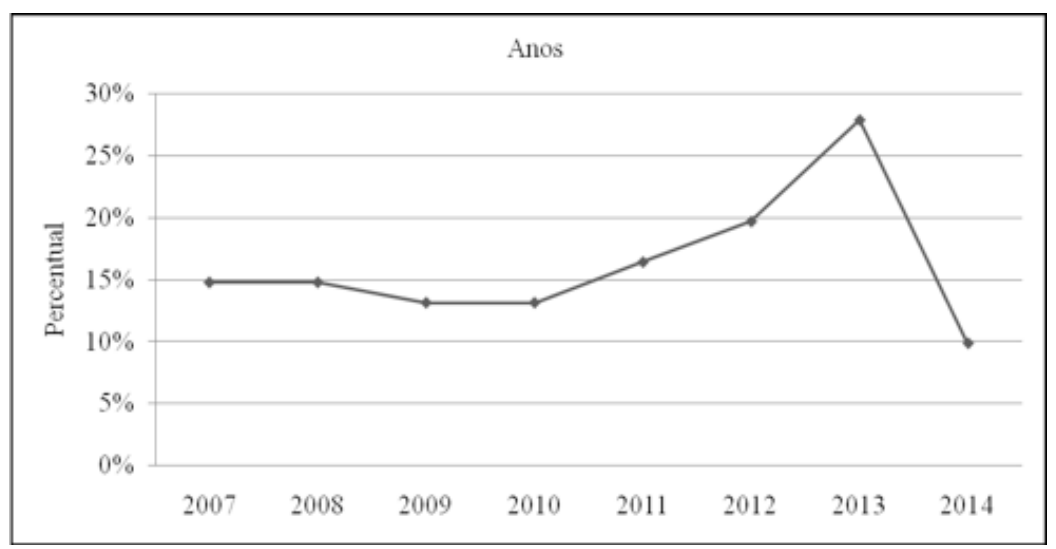

Fonte: Elaboração própria. 
O perfil dos idosos vítimas de suicídio no estado do Piauí corresponde a: sexo masculino (82\%), faixa etária de 60 a 70 anos, raça/cor parda $(63,9 \%) ;$ casados $(65,6 \%) ;$ com escolaridade entre 4 e 7 anos (32,8\%); aposentados (42,6\%); residentes no município de Teresina $(65,6 \%)$ (Tabela 1)

Tabela 1 - Características sociodemográficas dos óbitos por suicídio em idosos. Piauí, Brasil - 2007-2014 (N=61)

\begin{tabular}{|c|c|c|c|}
\hline \multicolumn{2}{|l|}{ Variáveis } & $\mathbf{n}$ & $\%$ \\
\hline \multirow[t]{3}{*}{ Faixa etária } & 60 a 70 & 33 & 54,0 \\
\hline & 71 a 80 & 21 & 34,5 \\
\hline & Acima de 80 & 7 & 11,5 \\
\hline \multirow[t]{2}{*}{ Sexo } & Feminino & 11 & 18,0 \\
\hline & Masculino & 50 & 82,0 \\
\hline \multirow[t]{5}{*}{ Raça/cor } & Branca & 19 & 31,1 \\
\hline & Preta & 3 & 4,9 \\
\hline & Amarela & 0 & 0 \\
\hline & Parda & 39 & 63,9 \\
\hline & Indígena & 0 & 0 \\
\hline \multirow[t]{5}{*}{ Estado civil } & Solteiro & 2 & 3,3 \\
\hline & Casado & 40 & 65,6 \\
\hline & Viúvo & 13 & 21,3 \\
\hline & Divorciado & 5 & 8,2 \\
\hline & União Estável & 1 & 1,6 \\
\hline Escolaridade & Nenhuma & 16 & 26,2 \\
\hline (Em anos de estudo & De 1 a 3 & 5 & 8,2 \\
\hline \multirow[t]{4}{*}{ concluído) } & De 4 a 7 & 20 & 32,8 \\
\hline & De 8 a 11 & 11 & 18,0 \\
\hline & 12 ou mais & 6 & 9,8 \\
\hline & Ignorado & 3 & 4,9 \\
\hline \multirow[t]{5}{*}{ Ocupação } & Aposentado & 26 & 42,6 \\
\hline & Lavrador & 14 & 23,0 \\
\hline & Comerciante & 5 & 8,2 \\
\hline & Dona do Lar & 4 & 6,6 \\
\hline & Outros & 12 & 19,7 \\
\hline \multirow[t]{5}{*}{ Município de residência } & Teresina & 40 & 65,6 \\
\hline & Beneditinos & 2 & 3,3 \\
\hline & São Miguel do Tapuio & 2 & 3,3 \\
\hline & União & 2 & 3,3 \\
\hline & Outros & 15 & 24,6 \\
\hline Total & & 61 & 100,00 \\
\hline
\end{tabular}

Fonte: Elaboração própria.

Os óbitos por suicídio em idosos no estado pesquisado ocorreram com maior frequência no município de Teresina (70,5\%); o domicílio foi o local de maior incidência em relação aos demais locais, correspondendo a $70,5 \%$; o meio mais utilizado foi enforcamento (78,7\%). (Tabela 2). 
Tabela 2 - Distribuição dos óbitos de idosos vítimas de suicídio, segundo município de ocorrência, local e meio utilizado. Piauí, Brasil - 2007-2014 (N=61)

\begin{tabular}{ll|c|c}
\hline Variáveis & & $\mathbf{n}$ & $\mathbf{\%}$ \\
\hline Município de ocorrência & Teresina & 43 & 70,5 \\
& São Miguel do Tapuio & 2 & 3,3 \\
& Altos & 1 & 1,6 \\
& Outros & 15 & 24,6 \\
& Domicílio & 43 & 70,5 \\
& Hospital & 9 & 14,8 \\
& Zona Rural & 3 & 4,9 \\
Mecorrência & Vias públicas & 2 & 3,3 \\
& Outros & 4 & 6,6 \\
& Lesão autoprovocada intencionalmente & 48 & 78,7 \\
& por enforcamento & & 8,2 \\
& Lesão autoprovocada intencionalmente & 5 & 8,2 \\
& por arma de fogo & & 1,6 \\
& Autointoxicação por exposição & 5 & 3,3 \\
& intencional a pesticidas & & \\
& Lesão autoprovocada intencionalmente & 1 & 100,00 \\
\hline
\end{tabular}

Fonte: Elaboração própria.

\section{Discussão}

Esta pesquisa verificou um aumento na taxa de óbitos a partir de 2010, atingindo o ápice no ano de 2013, e declínio em 2014. Este resultado corrobora os dados da literatura, na qual o suicídio na população idosa teve um aumento significativo nos últimos anos ${ }^{(9)}$. Apesar do declínio verificado no ano de 2014, é necessário atentar para a ocorrência do fenômeno.

Há uma convergência estatística que elucida o crescimento significativo da taxa de suicídio em idosos jovens com tendência de crescimento das taxas na população com idade entre 70 e 80 anos. Esta evidência também foi apontada em outros estudos realizados em estados do Nordeste com ênfase nos estados do Piauí, Ceará e Rio Grande do Norte ${ }^{(9)}$. Entre os idosos, é incomum histórico de tentativas prévias, já que planejam e utilizam meios letais de cometerem suicídio. Não obstante, é fundamental atentar para comportamentos autodestrutivos, autonegligência e valorizar indícios comportamentais e verbais de intenção suicida ${ }^{(10)}$.

Em relação ao gênero, observou-se que os homens são os que mais cometem suicídio e isto pode relacionar-se com a utilização de meios que apresentam maior poder de letalidade. O suicídio frequentemente está associado ao sexo masculino na maioria dos países, exceto na China, onde o fenômeno é mais prevalente em mulheres $^{(11)}$. O sexo, portanto, consiste em um fator de vulnerabilidade, pois a masculinidade está associada à ideia de que o homem é um ser autônomo, provedor da família, forte e viril que não expressa sentimentos ${ }^{(11-12)}$.

No que se refere à raça/cor, estudos internacionais apontam maior ocorrência em brancos, com exceção dos indígenas, que mostram riscos elevados em diferentes situações. Entretanto, nesta pesquisa, a raça/cor prevalente foi parda e branca, respectivamente. Este resultado é semelhante ao encontrado em outros estudos de âmbito nacional, que realçam homens pardos 
e mulheres brancas e pardas em proporção semelhante ${ }^{(13-14)}$.

Apesar de o casamento ser citado na literatura como fator de proteção, os idosos casados foram os que mais cometeram suicídio. Outras pesquisas revelaram a mesma situação identificada no Brasil, seguida da incidência de idosos viúvos. Desta maneira, enfatiza-se a necessidade de vigilância, inclusive em grupos considerados de baixo risco ${ }^{(11,15)}$. A maior incidência de óbito por suicídio em casados pode estar relacionada a fatores socioeconômicos, como dificuldades econômicas e desemprego, que induzem ao aumento do consumo de álcool e outras drogas, além de problemas no relacionamento familiar, sintomas depressivos, dentre outros ${ }^{(16)}$.

As questões socioeconômicas, como crises econômicas, desemprego e redução na renda pessoal, são fatores de risco importantes, principalmente para homens ${ }^{(17)}$. Nesta pesquisa foram elencados a baixa escolaridade e a aposentadoria. Os idosos com escolaridade de 4 a 7 anos concluídos ou nenhuma escolaridade foram, respectivamente, os que apresentaram maior índice de suicídio. Quanto maior o nível de escolaridade, menores são as probabilidades de dificuldades financeiras, já que este influi na estabilidade econômica, podendo propiciar às pessoas um envelhecimento de forma mais digna ${ }^{(10,18)}$.

No contexto sociocultural, a pessoa tem valor correspondente à sua produtividade. Dessa forma, o idoso pode ser visto como inútil em algumas situações. A incapacidade de prover a família financeiramente pode induzir a um sentimento de tristeza, angústia e deslocamento no ambiente em que vive o idoso, às vezes em decorrência da falta de comunicação e da relação afetivo-pessoal com a família. Os idosos tendem a se autoperceberem como estorvo, situação agravada com a perda de autonomia e dependência de cuidados relacionados às necessidades básicas ${ }^{(19)}$.

Ao considerar os municípios de residência e ocorrência dos óbitos, salienta-se que o suicídio de idosos na região Nordeste do Brasil pode estar associado com o fenômeno da migração do campo para a cidade. Isto porque, em tais situações de mudança, inúmeras pessoas demonstram algum grau de sofrimento pela ruptura de laços sociais, perda da cultura e dos vínculos, dificuldade de se adaptar aos costumes da vida urbana. Esses são fatores que contribuem, entre outros, para sua situação de vulnerabilidade ${ }^{(20)}$.

$\mathrm{Na}$ análise sociodemográfica de um estudo longitudinal irlandês sobre envelhecimento e ideação suicida, a região urbana também foi citada como a de maior incidência ${ }^{(18)}$. Neste estudo houve ainda suicídios em municípios divergentes daqueles nos quais os idosos residiam. Essa discordância em relação aos municípios pode estar relacionada ao fato de Teresina ser mais populosa ou, ainda, por ser município de referência na assistência em saúde no Estado, atrai imigrantes.

Nesta pesquisa, os óbitos ocorreram com maior frequência no domić́lio. Estudos ressaltam que a perda de valorização social e familiar pode resultar em isolamento. No contexto domiciliar, pode favorecer e até mesmo facilitar a execução do suicídio, já que os cuidadores podem ausentar-se para trabalho e atividades cotidianas ${ }^{(19)}$. Nos hospitais foi evidenciada a segunda maior taxa de óbitos. Isto pode estar relacionado ao fato de esses idosos, apesar de receberem atendimento de emergência, apresentarem quadro clínico irreversível.

No que se refere à efetivação do suicídio, os meios mais utilizados pelos idosos foram: enforcamento, seguido por arma de fogo e envenenamento/intoxicação exógena por uso de pesticidas/agrotóxicos. Os idosos tendem a usar métodos mais agressivos e letais, como enforcamento e uso de arma de fogo, este último principalmente em homens. Estudo em outros países evidencia que a perpetração do suicídio mais utilizada foi também o enforcamento, o estrangulamento e a sufocação utilizados por ambos os sexos ${ }^{(21)}$. Esta pesquisa ratifica os resultados desses estudos internacionais.

A ingesta de pesticidas é um método não violento comum no meio rural brasileiro ${ }^{(22)}$. Pesquisas nacionais relatam que o envenenamento/ intoxicação exógena é um meio para perpetrar suicídio comumente utilizado pelas mulheres. 
Merecem atenção especial os agrotóxicos, um dos meios mais utilizados no suicídio e nas tentativas de suicídio, quando se analisa a região Nordeste ou pessoas que vivem no meio rural. O uso de agrotóxico pode estar associado à frequente aplicação desses produtos na agricultura da região em estudo, ao conhecimento da população sobre seu alto poder tóxico e ao fácil $\operatorname{acesso}^{(10)}$.

No Brasil, não há uma base de dados que notifique a frequência e a distribuição das tentativas de suicídio. Isso contribui para a menor conscientização dos profissionais e dos gestores de saúde pública em relação ao impacto do comportamento suicida nos serviços de saúde ${ }^{(23)}$. É imprescindível que os profissionais da saúde conheçam e reconheçam as vulnerabilidades dos idosos, capacitem-se e colaborem com o desenvolvimento e aperfeiçoamento de políticas públicas voltadas para esse público.

Dentre as estratégias adotadas na prevenção do suicídio, estão a política de compra/venda de agrotóxicos, a instituição de políticas efetivas de desarmamento, uma política de saúde mental bem sedimentada, identificação e tratamento precoce, treinamento dos profissionais de saúde, acompanhamento e apoio comunitário após uma tentativa de suicídio ${ }^{(24)}$.

O declínio dos casos de suicídio no ano de 2014 pode relacionar-se à vertente preventiva local, por meio de mobilização social, ações em saúde e abordagem da mídia sobre o tema. Sugere-se estudos futuros que busquem fazer essa associação. O suicídio é cercado de inúmeros tabus, entretanto a complexidade do tema não deve inviabilizar a abordagem e, consequentemente, o planejamento de estratégias interventivas. Tendo em vista que o idoso tem se destacado nas estatísticas pelo número de suicídios, entende-se que os profissionais da atenção primária, como atuantes na promoção da saúde e na prevenção de doenças e agravos, devem estar qualificados para atender, identificar e individualizar as necessidades desse público.

O estudo trouxe como limitação a incompletude dos dados nas DO. Essa falha evidencia a necessidade de maior ênfase na qualificação dos profissionais e na implantação de estratégias que fortaleçam o entendimento de que a notificação de qualidade é necessária para garantir dados estatísticos fidedignos. O investimento tecnológico em sistemas de armazenamento de dados também pode garantir maior precisão e segurança.

\section{Conclusão}

A caracterização das vítimas de morte autoprovocada correspondeu a idosos com faixa etária de 60 a 70 anos, sexo masculino, raça parda, casados, baixa escolaridade, aposentados e residentes em zona urbana. O suicídio ocorreu predominantemente nos próprios domicílios e o enforcamento foi o meio mais utilizado. Apesar do declínio da estatística de suicídio no ano de 2014, reafirmou-se, neste estudo, que o fenômeno encontra-se em ascensão nos últimos anos neste grupo populacional.

Esta pesquisa possibilitou perceber que o declínio funcional vivenciado pelo idoso pode torná-lo vulnerável e modificar sua maneira de relacionar-se com as pessoas e com o mundo. Isso remete a pensar-se em novas perspectivas de, como profissionais, planejar a assistência ao idoso num âmbito biopsicossocial, valorizando a integralidade do cuidado. Há necessidade de refletir e elaborar estratégias que visem promover a saúde mental dos idosos na atenção primária com foco interdisciplinar.

Portanto, ratifica-se a necessidade de considerar a população idosa como grupo vulnerável ao suicídio por enforcamento em domicílio. É imperativo investimento em políticas públicas, principalmente na atenção primária, na qual os profissionais estão em contato direto com esse público, garantindo assistência e valorização dos aspectos relacionados à saúde mental, acesso a métodos terapêuticos e capacitação dos profissionais ante ao fenômeno. Possibilitar-se-á, assim, a identificação dos idosos com risco de suicídio e a adoção de métodos preventivos, respeitando a dignidade e promovendo qualidade de vida à pessoa idosa. 


\section{Colaborações:}

1. concepção, projeto, análise e interpretação dos dados: Adriana Vasconcelos Gomes, Prissilla Kalyne Bezerra Cardoso, Francisca Cecília Viana Rocha, Cláudia Maria Sousa de Carvalho e Magda Coeli Vitorino Sales;

2. redação do artigo e revisão crítica relevante do conteúdo intelectual: Adriana Vasconcelos Gomes, Francisca Cecília Viana Rocha e Magda Coeli Vitorino Sales;

3. aprovação final da versão a ser publicada: Adriana Vasconcelos Gomes, Prissilla Kalyne Bezerra Cardoso, Francisca Cecília Viana Rocha, Cláudia Maria Sousa de Carvalho e Magda Coeli Vitorino Sales.

\section{Referências}

1. United Nations. Department of Economic and Social Affairs. Population Division (2015). World Population Ageing. New York; 2015.

2. Fässberg MM, Cheung G, Canetto SS, Erlangsen A, Lapierre S, Lindner R, et al. A systematic review of physical illness, functional disability, and suicidal behaviour among older adults. Aging Ment Health [Internet]. 2016 [cited 2016 May 25];20(2):166-94. Available from: http://www.ncbi.nlm.nih.gov/ pmc/articles/PMC4720055/

3. World Health Organization. Preventing suicide: A global imperative. Genebra (CH); 2014.

4. Sérvio SMT, Cavalcante ACS. Retratos de autópsias psicossociais sobre suicídio de idosos em Teresina. Psicol cienc prof [Internet]. 2013 [cited 2015 Mar 10];33(n esp):164-75. Available from: http://www.scielo.br/scielo.php?script $=$ sci_ arttext\&pid=S1414-98932013000500016

5. Machado DB, Santos DN. Suicídio no Brasil, de 2000 a 2012. J bras psiquiatr [Internet]. 2015 Mar [cited 2016 Mar 19];64(1):45-54. Available from: http://www.scielo.br/scielo.php?pid=\$0047$20852015000100045 \&$ script $=$ sci_arttext $\&$ tlng=pt

6. Brasil. Ministério da Saúde. Portaria n. 1.876, de 14 de agosto de 2006. Institui diretrizes nacionais para prevenção do suicídio. Brasília (DF); 2006.

7. Brasil. Presidência da República. Lei n. 6.015, de 31 de dezembro de 1973. Dispõe sobre os registros públicos, e dá outras providências [Internet]. Brasília (DF); 1973 [cited 2015 Mar 25]. Available from: http://www.planalto.gov.br/ccivil_03/leis/ L6015compilada.htm

8. Brasil. Ministério da Saúde. Conselho Nacional de Saúde. Resolução n. 466, de 12 de dezembro de 2012. Aprova diretrizes e normas regulamentadoras de pesquisas envolvendo seres humanos [Internet]. Brasília (DF); 2012 [cited 2015 Mar 25]. Available from: http://bvsms.saude.gov.br/bvs/saudelegis/ cns/2013/res0466_12_12_2012.html

9. Pinto LW, Pires TO, Silva CMFP, Assis SG. Evolução temporal da mortalidade por suicídio em pessoas com 60 anos ou mais nos estados brasileiros, 1980 a 2009. Ciênc saúde coletiva [Internet]. 2012 ago [cited 2015 Mar 10];17(8):1973-81. Available from: http://www.scielo.br/scielo.php?pid=S1413$81232012000800008 \&$ script $=$ sci_abstract\&tlng=pt

10. Seleghim MR, Bellasalma ACM, Mathias TAF, Oliveira MLF. Caracterização das tentativas de suicídio entre idosos. Cogitare Enferm [Internet]. 2012 abr-jun [cited 2015 Dec 3];17(2):277-83. Available from: http://ojs.c3sl.ufpr.br/ojs/index. $\mathrm{php} /$ cogitare/article/view/25815

11. Ciulla L, Nogueira EL, Silva Filho IG, Tres GL, Engroff P, Ciulla V, et al. Suicide risk in the elderly: Data from Brazilian public health care program. J Affect Disord [Internet]. 2014 jan [cited 2015 May 10];152-154:513-6. Available from: http://www.sciencedirect.com/science/article/pii/ S0165032713004692

12. Meneghel SN, Gutierrez DMD, Silva RM, Grubits S, Hesler LZ, Ceccon RF. Suicídio de idosos sob a perspectiva de gênero. Ciênc saúde coletiva [Internet]. 2012 ago [cited 2015 Dec 10];17(8):1983-92. Available from: http:// dx.doi.org/10.1590/S1413-81232012000800009

13. Pinto LW, Silva CMFP, Pires TO, Assis SG. Fatores associados com a mortalidade por suicídio de idosos nos municípios brasileiros no período de 2005-2007. Ciênc saúde coletiva [Internet]. 2012 ago [cited 2015 Dec 10];17(8):2003-9. Available from: http://www.scielo.br/scielo.php?script=sci_ arttext\&pid=S1413-81232012000800011

14. Sena-Ferreira N, Pessoa VF, Boechat-Barros R, Figueiredo AEB, Minayo MCS. Fatores de risco relacionados com suicídios em Palmas (TO), Brasil, 2006-2009, investigados por meio de autópsia psicossocial. Ciênc saúde coletiva [Internet]. 2014 jan [cited 2016 May 13];19(1):115-26. Available from: http://www.scielo.br/scielo.php?script=sci_ arttext\&pid $=$ S1413-81232014000100115\&lng=en\& $\mathrm{nrm}=\mathrm{iso} \& \operatorname{lng}=\mathrm{pt}$ 
15. Sinyor M, Schaffer A, Streiner DL. Characterizing suicide in Toronto: an observational study and cluster analysis. Can J Psychiatry [Internet]. 2014 Jan [cited 2017 June 25];59(1):26-33. Available from: https://www.ncbi.nlm.nih.gov/pmc/articles/ PMC4079226/

16. Vidal CEL, Gontijo ECDM, Lima LA. Tentativas de suicídio: fatores prognósticos e estimativa do excesso de mortalidade. Cad Saúde Pública [Internet]. 2013 [cited 2015 Dec 2];29(1):175-85. Available from: http://www.scielosp.org/scielo.php?script=sci_ arttext\&pid=S0102-311X2013000500020

17. Turecki G, Brent DA. Suicide and suicidal behaviour. Lancet [Internet]. 2016 Mar [cited 2017 July 10];387(10024):1127-39. Available from: https://www.ncbi.nlm.nih.gov/pmc/articles/ PMC5319859/

18. Santini ZI, Koyanagi A, Tyrovolas S, Haro JM. The association of relationship quality and social networks with depression, anxiety, and suicidal ideation among older married adults: Findings from a cross-sectional analysis of the Irish Longitudinal Study on Ageing (TILDA). J Affect Disord [Internet]. 2015 July [cited 2016 May 13];179:134-41. Available from: http://www.ncbi. nlm.nih.gov/pubmed/25863909

19. Gutierrez DMD, Sousa ABL, Grubits S. Vivências subjetivas de idosos com ideação e tentativa de suicídio. Ciênc saúde coletiva [Internet]. 2015 jun [cited 2016 May 13];20(6):1731-40. Available from: http://www.scielo.br/scielo.php?script=sci_ arttext\&pid $=$ S1413-81232015000601731\&lng=en\& $\mathrm{nrm}=\mathrm{iso} \& \operatorname{lng}=\mathrm{en}$

20. Sousa GS, Silva RM, Figueiredo AEB, Minayo MCS, Vieira LJES. Circunstâncias que envolvem o suicídio de pessoas idosas. Interface (Botucatu) [Internet]. 2014 [cited 2015 Dec 10];18(49):1-13. Available from: http://www.scielo.br/scielo.php?script $=$ sci_ arttext\&pid=S1414-32832014000200389

21. Pinto LW, Assis SG, Pires TO. Mortalidade por suicídio em pessoas com 60 anos ou mais nos municípios brasileiros no período de 1996 a 2007. Ciênc saúde coletiva [Internet]. 2012 ago [cited 2015 Dec 10];17(8):1963-72. Available from: http://www.scielo.br/scielo.php?script=sci_ arttext\&pid=S1413-81232012000800007

22. Sun SH, Jia1 CX. Completed suicide with violent and non-violent methods in rural Shandong, China: a psychological autopsy study. PLoS One [Internet]. 2014 Aug [cited 2017 June 25];9(8):e104333. Available from: https://www.ncbi.nlm.nih.gov/ pmc/articles/PMC4128761/

23. Rios MA, Anjos KF, Meira SS, Nery AA, Casotti CA. Completude do sistema de informação sobre mortalidade por suicídio em idosos no estado da Bahia. J Bras Psiquiatr [Internet]. 2013 [cited 2015 Dec 3];62(2):131-8. Available from: http://www.scielo.br/scielo.php?script $=$ sci_ arttext\&pid=S0047-20852013000200006

24. Fleischmann A, Arensman E, Berman A, Carli V, Leo D, Hadlaczky G, et al. Overview evidence on interventions for population suicide with an eye to identifying best-supported strategies for LMICs. Glob Ment Health [Internet]. 2016 Feb [cited 2017 June 25];3:e5. Available from: https://www.ncbi. nlm.nih.gov/pmc/articles/PMC5314741/

Recebido: 29 de março de 2018

Aprovado: 22 de outubro de 2018

Publicado: 26 de dezembro de 2018

A Revista Baiana de Enfermagem utiliza a Licença Creative Commons - Atribuição-NãoComercial 4.0 Internacional.

https://creativecommons.org/licenses/by-nc/4.0/

Este artigo é de acesso aberto distribuído sob os termos da Licença Creative Commons (CC BY-NC).

Esta licença permite que outros remixem, adaptem e criem a partir do seu trabalho para fins não comerciais. Embora os novos trabalhos tenham de lhe atribuir o devido crédito e não possam ser usados para fins comerciais, os usuários não têm de licenciar esses trabalhos derivados sob os mesmos termos. 